\title{
AN ASSESSMENT OF NATURAL LANGUAGE ROBUSTNESS AND HOW IT RELATES TO NATURAL LANGUAGE SUSTAINABILITY: A FUZZY ECOLOGICAL APPROACH TO THE LIVES OF NATURAL LANGUAGES
}

\author{
OCENA TĘŻYZNY JĘZYKÓW NATURALNYCH \\ I JEJ ZWIĄZKU Z ICH PRZEŻYWALNOŚCIĄ: \\ ROZMYTE PODEJŚCIE EKOLOGICZNE DO KWESTII ŻYCIA \\ JĘZYKÓW NATURALNYCH
}

\author{
STANISEAW PUPPEL
}

\begin{abstract}
All living natural languages may be characterized in terms of their overall 'robustness'. The concept may be defined as relating directly to the health/vitality / wellbeing (i.e. sustainability) of these languages. In the paper, an attempt is made to further define the concept in terms of a set of parameters as well as to show that natural languages differ as to the degree of their overall robustness.
\end{abstract}

Stanisław Puppel, Uniwersytet im. Adama Mickiewicza w Poznaniu, Poznań - Polska.

A natural language may be characterized in terms of its 'robustness'. The concept may be defined as an interplay of a number of selected parameters and their fuzzy values contributing jointly to the language's overall ‘health/vitality/wellbeing' (see Puppel, 2007; 2009). Natural language robustness (hence NLR) is a single major factor responsible for the fairing of a given NL against other natural languages in the Natural Language Global Arena (hence NaLGA) where they compete and/or collaborate with each other under regular contact conditions. In assessing the degree of robustness of a natural language, the following two groups of parameters, internal and external, should be distinguished and taken into consideration. However, since they cannot be measured precisely by means of a decimal system, only fuzzy values may be postulated. That is why a fuzzy approach to the problem of NLR is undertaken here. The selected set of parameters presented below are further shown as producing a set of generic outcomes illustrating the significance of the NLR factor for natural language sustainability (survivability). 


\section{The Internal Parameters of NLR}

(i.e. those parameters which may be defined as the ones which characterize the internal state/dynamism of any NL at a given point of time)

NLs characterized in terms of the types of modalities used in current communicative practice:

1. Audio-vocal modality (AVo):

- regional diversification of spoken (oral) language including: the presence, number and size of regional dialects as some kind of 'breeding loci' for a national (standard) language as a dialectally polymorphic phenomenon (i.e. the presence of regional dialects versus standard dialect),

- the presence and size of culture-specific non-verbal communicative resources such as culture-determined paralanguage (cf. Poyatos, 1993).

2. Visual-tactile modality (ViT):

- the presence, degree of diversification and size of means of written language including: literature, poetry, printed grammars, legal documents, religious documents, scientific writings, daily press, journals, encyclopedias, dictionaries, other books, other printed materials, all contributing to a given language's internal degree of 'graphic affluence',

- the presence and size of culture-specific non-verbal resources comprising: culture-specific elements of non-verbal communication such as gestures, facial expressions, and body postures,

- the presence and use of a local sign language (e.g. American Sign Language, Nicaraguan Sign Language, etc.).

3. AVo and ViT modalities combined (the so-called hybrid audio-visual order of communication, $\mathrm{AVi}$ ):

- the presence, degree of diversification and size of audio-visual means of communication including: feature films, documentaries, TV programs, etc., usually contributing jointly to the development and strengthening of the standard dialect. It should be noted at this point that not all local language communities have at present reached that advanced stage of development of hybridity of communicative modalities and many still remain in their spoken stage (see point 1 above; also cf. Grimes, 2000).

Outcome nr 1: a natural language which has developed and which uses all three modalities in the audio-visual order of communication may be defined as a modality-complete language which has thus reached the highest degree of language and non-language resource combination and intensification. Such a language is also characterized as a robust language. 
NLs characterized in terms of their communicative fitness further determined by the local environment (i.e. their use in the environmentalcommunicative niches):

4. The diversification and size of the NL resources is dependent on the particular niche(s) in which it is used. The following communicative niches may be distinguished:

- the daily routine and general culture niche in which the majority of native communicators 'reside' most of the time of their daily verbal and non-verbal interactions with other communicators,

- the professional language niche used in the workplace and study place (including permanent and transitory sociolects, i.e. the sociocultural diversification of spoken and written forms of a NL, also the presence of sophisticated professional language resources organized in numerous professional sub-niches),

- the citizenship niche used in the legal (i.e. constitutional and juridicial) manifestations of the NL resources.

Outcome nr 2: a natural language which has developed its resources in all the three niches is a full-niche and robust NL and thus a particular human communicating agent (hence HCA) who has gained control over these niches may be regarded as a complete (hence more robust) communicator in terms of individual language resource control.

\section{The External Parameters of NLR}

(i.e. those parameters which may be defined as the ones which characterize the external state/dynamism of any NL at a given point of time)

NLs characterized in terms of their territorial range in the coreperiphery model of their occurrence and subsequent population mobility:

5. The size of the area of the core of the NL habitat (e.g. determined in $\mathrm{km}^{2}$ ):

- 'large core area' NLs (e.g. Chinese, English, Spanish)

- 'small core area' NLs (e.g. Lithuanian, Latvian, Hungarian),

- 'coreless' NLs (e.g. Romani).

The population mobility which results from the above distinctions may include the following types:

- a population may stay exclusively within the core of the primary habitat, that is, it prefers not to disperse (e.g. the modern Norwegian population in its original habitat). Historically speaking, such a strong preference for the core may result in the establishment of coredominant languages, either one-core dominant NLs (e.g. Polish), or many-core dominant NLs (such as English, Spanish, Portuguese), 
- a population may stay within the core habitat while also being open towards and exercising immigration (e.g. the Polish population) thus exercising the so-called centrifuge effect (see point 7 below).

Outcome nr 3: a natural language which is characterized by the occurrence in and domination of one or more cores may be regarded as one which demonstrates a greater degree of robustness.

6. Geographical diversification of the area of the core of the NL habitat (naturally combined with the size of the area of the core) both contribute to a greater wealth of a given NL generated by the potential occurrence of a larger number of regional dialects and a larger population using the language. The latter is rendered most efficiently by NL 'weight', expressed by the term 'linguomass/glottomass' understood as follows: the bigger is the population using a particular NL, the bigger is its linguomass/glottomass (i.e. the number of native communicators) and the bigger is its expressive capacity. The natural geographical diversification may include: lowlands, mountains, coastal regions, forested areas, desert regions and other natural dividers (see e.g. Coe et al., 2007).

Outcome nr 4: a natural language which is characterized by a larger territorial range (size) of its core habitat and subsequent greater geographical diversification may tend to possess a bigger number of regional dialects whose presence overall is an indicator of a NL's greater degree of robustness.

NLs characterized in terms of spatial 'navigation' effects:

7. The centrifuge effect where there occurs readiness of the members of a given linguistic community to outmigrate, that is, to move to regions located outside the primary core habitat. There is readiness to create the NL periphery as an extension of the core of the habitat via population dispersion. The centrifuge effect is more physical in nature and consists in dispersing members of a linguistic community and thus leading to the weakening of its 'linguomass' in the core.

8. The magnet effect where there occurs readiness of the members of a given linguistic community to 'absorb' the entire outer social and linguistic space into the core of its habitat by whatever means available to the community. This also includes the accompanying mental magnification of signification and resultant imposition of a receiving language over the arriving language(s) such that, as a result of the culture-specific magnet effect, the receiving language is perceived by the 'guest' communicators as sufficiently attractive and thus worthy 
of learning. The magnet effect is more mental in nature and consists in withholding the magnet-driven population within the core of the receiving community both for reasons of identification (i.e. a sense of higher value) and economic stability.

Outcome nr 5: a natural language which is characterized by a greater tendency to disperse may be defined as weaker and thus less robust, while a NL which tends to attract other languages while being kept within the core habitat may, in turn, be regarded as a more robust language.

NL typology characterized in terms of the demographic factor of overall population 'weight', that is, linguomass/glottomass/expressive capacity:

The following tentative typology of NLs with regard to their population 'weight' may be proposed:

- 'heavy' NLs (with over 100 million native users: e.g. Mandarin Chinese, English, Hindu, German, French),

- 'semi-heavy' NLs (below 100 million native users: e.g. Urdu, Punjabi, Korean, Italian),

- 'light' NLs (below 50 million native users: e.g. Polish, Ukrainian, Persian, Malay),

- 'semi-light' NLs (below 10 million native users: e.g. Czech, Belorussian, Swedish, Slovak),

- 'endangered' NLs (below 1 million native users: e.g. Kui, Garo, Mizo, Munda),

- 'near-extinct' NLs (below 10,000 native users: e.g. Inpui Naga, Gahri, Thulung, Kota, Toda),

- 'extinct' NLs (with either few old living users or no users at all: e.g. Godwari, Khamyang, Great Andamanese, Weyto; see e.g. Grimes, 2000).

Outcome $\mathrm{nr}$ 6: a natural language which is characterized by greater weight, that is, by a greater linguomass/glottomass/expressive capacity may be defined as a more robust NL.

NL typology characterized in terms of the urban-rural ratio:

It is for the first time in the history of mankind that the world population has crossed the 50 per cent mark and is currently more urban than rural (see UN World Urbanization Prospects, 2009). The fact that the ratio urban:rural is now very much in favour of the rapidly growing city population which, as estimated by the above UN Report, is expected to increase steadily and considerably by the year 2050. This fact is also expected to exert a lasting influence on the emerging type of communicators, the urban 
communicators, who may soon dominate the linguistic landscape and who may be characterized in the following way:

- the urban communicator participates in a relatively denser network of HCAs as potential communicators and in a potentially greater number of communicative events, with big-sized AVo and ViT modalities involved,

- the urban communicator has relatively easier access to maximally diversified language resources,

- the urban communicator is exposed to and participates in a relatively bigger number of communicative encounters, both planned and random, with the involvement of both the AVo, ViT and hybrid AVi modalities,

- the urban communicator makes relatively higher-quality, more diversified and more frequent contributions to the common language resources, both local and standard,

- overall, the urban communicator makes a greater and more intense contribution to the maintenance and development of the size of language resources within the linguomass/expressive capacity and across all the communicative niches thus creating a greater language demandsupply chain (network) than the rural counterpart.

Outcome nr 7: a natural language which is characterized by a greater urbanization ratio and which is used by a greater number of urban communicators who jointly contribute to the NL's greater degree of robustness is licensed to increase that language's survivability.

Summing up the above discussion, one may state that natural languages differ considerably in the degree of their robustness as an outcome of an interplay of both internal and external parameters. Moreover, a higher degree of robustness of any existing NL is assumed to directly contribute to the language's health and power which, subsequently, increases its survivability (sustainability) in the long run vis-à-vis other natural languages. Thus, low or high fuzzy values that a set of robustness parameters a particular NL may obtain are assumed to be directly responsible for the fate of that language in the NaLGA.

\section{References}

C o e, N.M., P.F. K e 11 y and H.W.C. Y e u n g. 2007. Economic geography: a contemporary introduction. Oxford: Blackwell.

Gr i m e s, B.F. (ed.). 2000. Ethnologue: languages of the world. 14th ed. Dallas, TX: Summer Institute of Linguistics. 
P o y a t o s, F. 1993. Paralanguage: a linguistic and interdisciplinary approach to interactive speech and sounds. Amsterdam: John Benjamins.

P u p p e 1, S. 2007. „Tężyzna języków naturalnych”. In: Chałacińska-Wiertelak, H. (ed.). Dyskurs wielokulturowy. Prace Humanistycznego Centrum Badań. Łódź: Drukarnia i Wydawnictwo PIKTOR. 7-15.

P u p p e 1, S. 2009. "The protection of natural language diversity - fancy or duty?". Scripta Neophilologica Posnaniensia. Tom X. 97-109.

P u p p e 1, S. 2011. "The universal natural language preservation mechanism: an ecological approach". In: Puppel, S. (ed.). Transkomunikacja. W strone sprofilowania przestrzeni publicznej jako wielopłaszczyznowej przestrzeni komunikacyjnej. Poznań: Zakład Graficzny UAM. 91-99.

United Nations Department of Economic and Social Affairs/Population Division. World Urbanization Prospects: The 2009 Revision. 
